\title{
NORTH-SOUTH ASYMMETRY OF ULTRA-LOW-FREQUENCY OSCILLATIONS OF EARTH'S ELECTROMAGNETIC FIELD
}

\author{
A.V. Guglielmi \\ Institute of Physics of the Earth RAS, \\ Moscow, Russia, guglielmi@mail.ru \\ B.I. Klain \\ Borok Geophysical Observatory of IPE RAS, \\ Yaroslavl Region, Borok, Russia, klb314@mail.ru
}

\author{
A.S. Potapov \\ Institute of Solar-Terrestrial Physics SB RAS, \\ Irkutsk,Russia,potapov@iszf.irk.ru
}

\begin{abstract}
In the paper, we present the result of an experimental study of north-south asymmetry of ultralowfrequency electromagnetic oscillations IPCL. This study is based on observations made at Mirny Observatory (Antarctica). IPCLs are excited in the dayside sector of the auroral oval in the range 3-10 min periods and represent one of the most powerful types of oscillations of Earth's magnetosphere. These oscillations were discovered in the 1970s during IPhE AS USSR polar expeditions organized by Prof. V.A. Troitskaya. We have shown that IPCL activity in Mirny depends on the inclination (north-south asymmetry) of interplanetary magnetic field (IMF) lines to the plane of the geomagnetic equator be-
\end{abstract}

fore the front of the magnetosphere. The result suggests a controlling exposure of IMF on the magnetospheric oscillations and gives rise to the hypothesis that IPCL are forced oscillations of a nonlinear dynamical system whose major structural elements are dayside polar cusps. The paper is dedicated to the memory of Professor V.A. Troitskaya (1917-2010).

Keywords: magnetosphere, solar wind, geomagnetic pulsations, interplanetary magnetic field, Antarctica.

\section{INTRODUCTION}

Systematics of geomagnetic pulsations was developed in the early 1960s [Troitskaya, 1964]. It is still successfully used for describing ultralow-frequency (ULF) electromagnetic oscillations of Earth's magnetosphere (see, for example, overviews [Troitskaya, Guglielmi, 1967; Troitskaya, Guglielmi, 1969; Guglielmi, Troitskaya, 1978; Guglielmi, 1989; 2007], and monographs [Jacobs, 1970; Guglielmi, Troitskaya, 1973; Nishida, 1978; Guglielmi, 1979; Guglielmi, Pokhotelov, 1996]). The classification is based on the morphological principle, and a binomial nomenclature was introduced. Pulsations of all types are divided into two classes: Pc (pulsations continuous) and Pi (pulsations irregular). A distinctive feature of the class is the oscillogram shape. The type is symbolized as Pc $N(N=1-5)$ or Pi $N(N=1-$ 2 ), where the number $N$ is the subrange number of the general range of ultra-low frequencies. The Pc class was originally allocated a range of periods from 0.2 to $600 \mathrm{~s}$; and the class $\mathrm{Pi}$, from 1 to $150 \mathrm{~s}$. Soon, however, it became clear that the Pi range should be expanded, and, accordingly, the Pi3 type should be added to denote irregular oscillations in the range 150-600 s. In this paper, we deal with magnetospheric pulsations of this very type.

Observations show that according to morphological characteristics ULF oscillations are clearly subdivided into numerous types or, more accurately, subcategories. We focus on the subcategory Pi3 that in literature is often abbreviated to IPCL (irregular pulsations continuous long). This abbreviation indicates that pulsations of this subcategory, while being irregular, are continuous. In these cases, after a number denoting a subrange number, the letter $\mathrm{C}$ is put for greater clarity. For example, the Pi1 subcategory having a non-system name auroral agitation is sometimes referred to as Pi1C [Troitskaya, 1961; Kalisher, 1975]. Similarly, we could use the designation Pi3C instead of IPCL. This is an accurate indication to IPCLs within the systematics of ULF oscillations, the principles of which were formulated by prof. V.A. Troitskaya. We, however, retain the abbreviation IPCL, following the tradition established in recent years in geomagnetism.

Let us give some information on the IPCL morphology. For more detail, see [Bolshakova et al., 1974; Troitskaya, Bolshakova, 1977, 1988; Troitskaya, 1985; Klain et al., 2008]. Figure 1 presents an oscillogram of oscillations recorded at Mirny Observatory (corrected geomagnetic coordinates $\Phi=-76.93^{\circ}, \Lambda=122.92^{\circ}$ ). The oscillation spectrum is wide; characteristic periods are $3-10 \mathrm{~min}$. The amplitude of oscillations is variable with typical values from 5 to $10 \mathrm{nT}$ and sometimes reaches many tens of nT. Seasonal activity of IPCL is characterized by a summer maximum. On average, the amplitude increases with solar wind velocity. The oscillations usually occur at low and moderate geomagnetic activity $\left(K_{\mathrm{p}}=0-3\right)$.

The most constant distinctive feature of IPCL is that, as already mentioned, the pulsations are observed in the dayside auroral oval. This very property gave us the idea of formulating a hypothesis on possible mechanism of IPCL excitation and suggested testing the hypothesis by searching for the north-south asymmetry of the oscillations. The matter seems to be as follows. If there exists a north-south asymmetry that depends on the orientation of the interplanetary magnetic field (IMF) lines, then this new, previously unknown property of IPCL argues for our hypothesis on the excitation mechanism. 


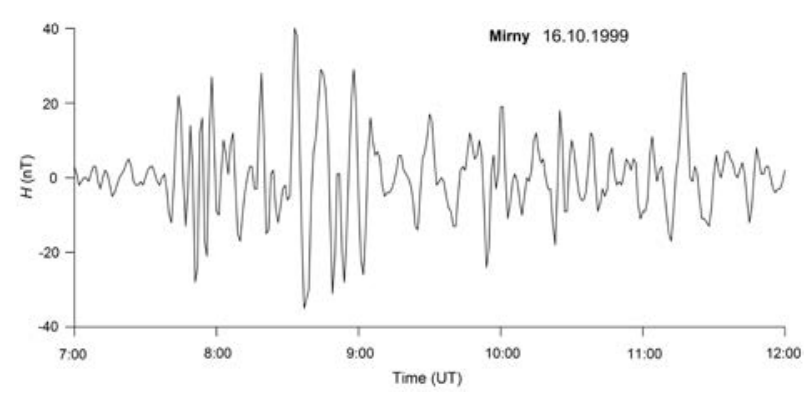

Figure 1. Magnetogram of ULF IPCL registered at Mirny Observatory (Antarctica) on October 16, 1999

In Section 1, we put forward an original hypothesis on IPCL excitation. In Section 2, we propose a method for testing the hypothesis. In Section 3, we make a critical check. Alternative excitation mechanisms are discussed in Section 4; in Conclusion, we summarize our IPCL study.

\section{IPCL EXCITATION SCENARIO}

There are two structural elements at the dayside magnetopause. They are located in high latitudes approximately symmetrically to the plane of the geomagnetic equator. These elements are called cusps [Yamauchi et al., 1996]. Through the cusps, hot solar wind plasma penetrates deep into the magnetosphere. We are interested, however, in ULF oscillations, not in plasma flows. In this regard, we call attention to two properties of the cusps, which are extremely important from the viewpoint of IPCL physics. The former property is that a cusp is projected by geomagnetic field lines onto the zone of the most frequent occurrence and maximum amplitude of IPCL.

The latter property is less obvious. A cusp seems to be a specific attractor for surface MHD waves excited at the magnetopause as a result of the Kelvin-Helmholtz instability. In other words, a cusp is most likely to have focusing properties. This is indicated by the following qualitative reasoning. In the cusp, the magnetic field $B$ is reduced and plasma density $\rho$ is increased. Thus, there is a minimum Alfvén velocity in the cusp: $c_{\mathrm{A}}=B / \sqrt{4 \pi \rho}$. According to the ray path theory of MHD waves, rays in inhomogeneous magnetoactive

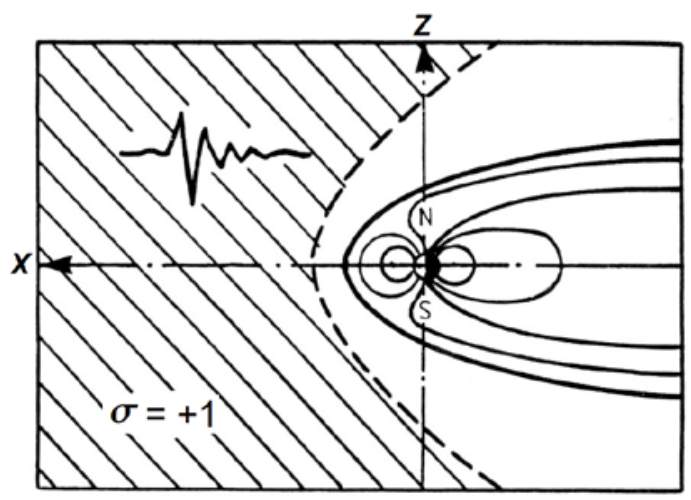

plasma bend toward a decrease in $c_{\mathrm{A}}$ [Guglielmi, 1985]. These general considerations do not, of course, solve the problem of surface wave propagation near the cusp at a proper physical-mathematical level, but make our assumption of the cusps' focusing properties quite plausible.

Here, we need to make one clarification. When the magnetosphere is crossed by the plane of the noon meridian, the magnetopause represents a curved line convex toward the Sun, and the two cusps look like earthward edges. In a three-dimensional space, the cusp resembles a cleft oriented in the west-east direction rather than a funnel as one might think taking the common interpretation of the term. Therefore, if we consider the focusing properties of the cusp, we must draw an analogy not with a spherical lens, but with a cylindrical lens. So, it stands to reason that the energy of surface waves flows into the cusp. But the cusp is projected onto Earth's surface just where IPCL occur. It is quite natural to assume that cusp pulsations excite Alfvén waves which transfer oscillation energy along geomagnetic field lines to Earth's surface. When moving from the cusp to its projection on the ionosphere, the oscillation amplitude increases.

The ray path theory holds that the amplitude of magnetic field oscillations is proportional to $\rho^{1 / 4}$ [Lundin, Guglielmi, 2006]. This gives us an approximate estimate of an increase in amplitude by an order of magnitude when passing from the cusp to Earth.

An interesting picture is emerging. There is a dynamical system whose elements are cusp and geomagnetic field lines projecting the cusp onto Earth's surface. We observe oscillations of the system in the form of IPCL. As in many other similar cases, a key question arises: what do we observe - self-oscillations or forced oscillations of the dynamic system? The question cannot be solved from a priori considerations since our system is not autonomous. We need to design a decisive experiment that would clarify the situation. However, the above question may appear to have no unambiguous answer, therefore we simplify our task and look for an answer to the question in a weaker formulation. Namely, let us try to experimentally find out whether the variable driving forces influence the IPCL excitation mode.

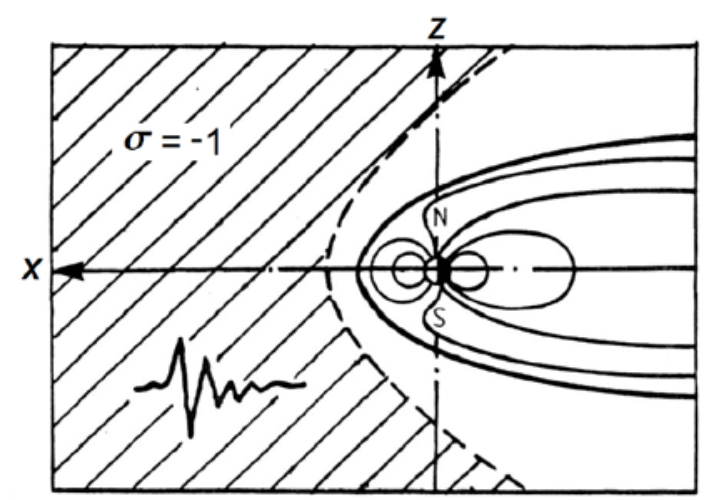

Figure 2. Cross-sections of the magnetosphere by the plane of the noon meridian at two IMF orientations [Guglielmi, Potapov, 2017] 


\section{TEST PROCEDURE}

So, we have no reliable information about the IPCL excitation mechanism and cannot perform physical and mathematical modeling of the oscillatory process simulating IPCL because the structure of our dynamic system is known only in the most general terms. The experimentally discovered phenomenon of intermittency in the IPCL excitation mode (Klain et al., 2008) suggests that we are most likely dealing with a strongly nonlinear dissipative oscillatory system. However, composition and operation of the system are in effect unknown to us; therefore it is quite appropriate to use the concept "black box" with output and input [Ashby, 1959]. At the output of our "black box", we observe IPCLs. Let us try somehow to "influence" the input so that to get an idea about the system operation mechanism from the reaction at the output. As the input it is natural to consider the mouth of the cusp.

Here we should recall that there is a significant difference between laboratory experiments of this kind and experiments in the magnetosphere. In a laboratory, an experimenter can voluntarily send some probing pulses to the input and judge on operation of the dynamic system by the reaction at the output; however, with rare exception, it is impossible in the magnetosphere. We have only one thing left: guided by general knowledge of the structure and dynamics of near-Earth space, to expect an external impact on the input of the system for a critical test of a hypothesis by analyzing the phenomena observed at the output of the system. Our idea of testing the hypothesis about IPCLs as forced pulsations is to partially control the effect of external forcing on the mouth of the cusp, using data on IMF orientation.

We have presented the driving forces as surface MHD waves for which the cusp is a cylindrical lens. But the cusp has focusing properties also for volume MHD waves existing permanently before the near-Earth shock front, penetrating into the transition layer, and reaching the magnetopause. We know that the mode of radiation of the magnetopause by volume waves depends on IMF orientation [Guglielmi et al., 2015]. We use this knowledge to find the answer to the question posed at the end of the previous section.

Let us explain the foregoing with the aid of Figure 2. It schematically shows two projections of the magnetosphere in the plane of the noon meridian at two different orientations of IMF lines. The cusps are located between closed and open geomagnetic field lines. The dashed line represents the near-Earth shock front. Slanting lines are IMF lines. Between the front and the IMF line tangent to the front, there is a so-called foreshock a specific region of near-Earth space in which strong fluctuations of the electromagnetic field are permanently excited in the frequency range from millihertz to several kilohertz. The excitation is caused by beam instability of interplanetary plasma interacting with a shock front [Russell, Hoppe, 1983]. In Figure 2, the fluctuations are schematically shown in the form of wave packets. The idea of the hypothesis testing method is that the impact on a cusp exerted by fluctuations penetrating through the shock front and reaching the magnetopause can be weakened or strengthened depending on IMF orientation.

Let us introduce a dichotomous variable $\sigma=\operatorname{sign}\left(B_{x} B_{z}\right)$, taking the values $\sigma=-1$ for $B_{x} B_{z}<0$ and $\sigma=+1$ for $B_{x} B_{z}>0$, where $B_{x}$ and $B_{z}$ are IMF components in the coordinate system shown in Figure 2. We see that the field lines are slanting to the north at $\sigma=+1$ and to the south at $\sigma=-1$. Hence, the asymmetry of the foreshock position relative to the equatorial plane changes qualitatively as the sign of $\sigma$ changes. It is obvious that electromagnetic waves excited in the foreshock region generally penetrate into the Northern (Southern) Hemisphere at $\sigma=+1(\sigma=-1)$.

These considerations suggest applying the hypothesis testing procedure: we should experimentally test whether activity of IPCLs, recorded, for example, at an observatory in the Southern Hemisphere, is actually higher at $\sigma=-1$ than at $\sigma=+1$. In the next Section, we describe the result of the test, using IPCL observations from Mirny Observatory located in the Southern Hemisphere.

\section{NORTH-SOUTH ASYMMETRY OF IPCLs}

First we recall the eight-dimensional control parameter space (CPS), which has been introduced in [Guglielmi et al., 2015] for the systematic study of the IMF role in forming the excitation mode of magnetospheric ULF oscillations. The visualization of IPCL data in CPS is an important part of qualitative study of experimental data. We use a visualization method based on the location of points in a space of smaller dimensionality [Davison, 1988].

Figure 3 exemplifies the visualization of events in the three-dimensional space formed by IMF components. The event is the IPCL observation time interval at Mirny Observatory. The richest archive of records of ULF oscillations, recorded at Mirny during Antarctic expeditions of the Institute of Physics of the Earth of the USSR Academy of Sciences organized by V.A. Troitskaya, is stored at Borok Observatory. One of the authors of the article (B.I. Klain) has used the archival materials, as well as the WDC database [http://www.wdcb.ru/stp/index.html] for constructing Figure 3.

Selection, processing, and analysis of events have been carried out as follows. By analyzing magnetograms for 1999 , we selected events recorded within \pm 2 $\mathrm{h}$ relative to the local geomagnetic noon, which corresponds to $8 \mathrm{~h}$ UT, with the additional restriction that geomagnetic activity indices satisfy the conditions $K_{\mathrm{p}}<3$, Dst $>-30 \mathrm{nT}$, i.e. the magnetosphere is in relatively quiet state. Altogether we have selected 95 events. Each event is associated with hourly averages of the three IMF components listed in the OMNI2 catalog [http://omniweb.gsfc.nasa.gov/ow.html].

In addition, each event was analyzed to determine a dominant period and hourly average oscillation amplitude. To do this, we analyzed dynamic spectra and de- 


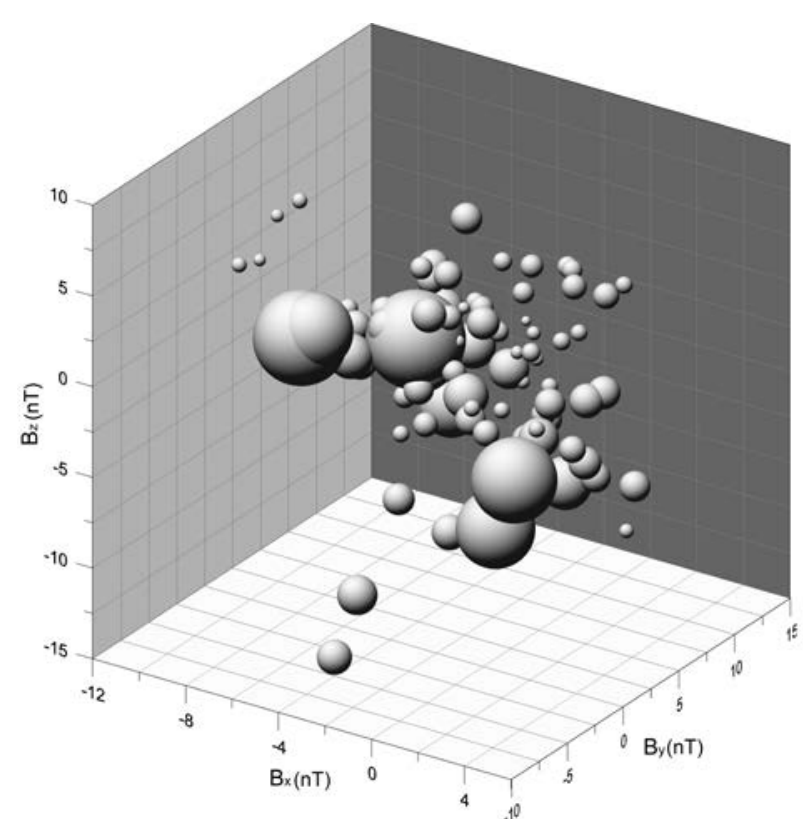

Figure 3. Distribution of events in the three-dimensional control parameter space formed by IMF components. Radii of bubbles are proportional to the IPCL amplitude

termined envelopes of signal amplitudes, using the Hilbert transform. As a result, it turned out that the period average for the sample was $3.5 \mathrm{~min}$, and the amplitude was 6 nT.As shown in Figure 3, the oscillation amplitude (it is proportional to the bubble radius) is quite variable. Mentally projecting centers of the bubbles on a horizontal plane, we see that in most cases the oscillations are observed when $\operatorname{sign}\left(B_{x} B_{y}\right)<0$. This correlates well with the most typical configuration of IMF lines, twisted into the Parker spiral due to the solar rotation [Parker, 1965]. Thus, Figure 3 endures an elementary test for the presence of information content in the threedimensional subspace of CPS.

Now consider Figure 4. It looks like Figure 3 except that instead of $B_{y}$ we use the azimuthal interplanetary electric field component $E_{y}$; and instead of $B_{z}$, the argument $B_{x} B_{z}$ of the function $\sigma$ introduced in Section 2. We see that IPCLs occur in the Southern Hemisphere more often at $\sigma=-1$ than at $\sigma=+1$. This qualitatively agrees well with our hypothesis about the existence of the north-south asymmetry of IPCL excitation. There is no doubt that in the Northern Hemisphere IPCLs occur more often at $\sigma=+1$ than at $\sigma=-1$. (Nevertheless, we plan to verify this statement, using observations of ULF oscillations made at observatories located in Greenland.)

To quantify the reliability of existence of the northsouth asymmetry, we have done a comparative analysis of IPCL amplitude distributions at $\sigma=-1$ and $\sigma=+1$.

Both the distributions are shown in Figure 5. Gaussian approximations of the distributions are shown by solid lines. The range, average amplitude, and dispersion are $21 \mathrm{nT}, 6.4 \mathrm{nT}$, and $24.3 \mathrm{nT}^{2}$ at $\sigma=-1$, and 12 $\mathrm{nT}, 5.4 \mathrm{nT}$, and $7.68 \mathrm{nT}^{2}$ at $\sigma=+1$. According to the Ftest, the difference between two samples is statistically significant at a significance level of 0.05 . In other

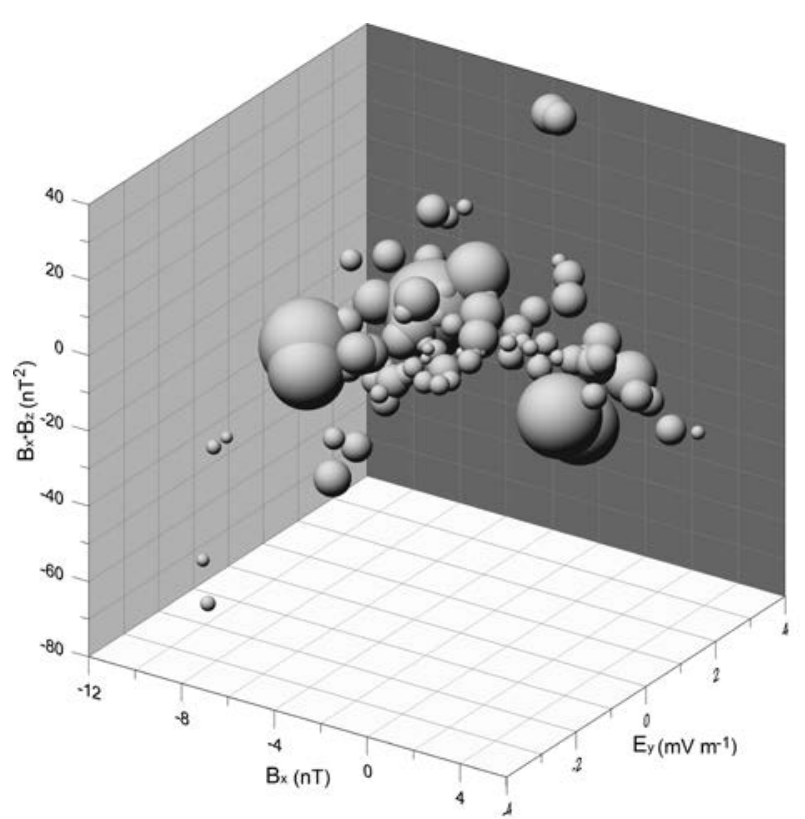

Figure 4. Distribution of events in the three-dimensional space formed by heterogeneous components of the general space of control parameters

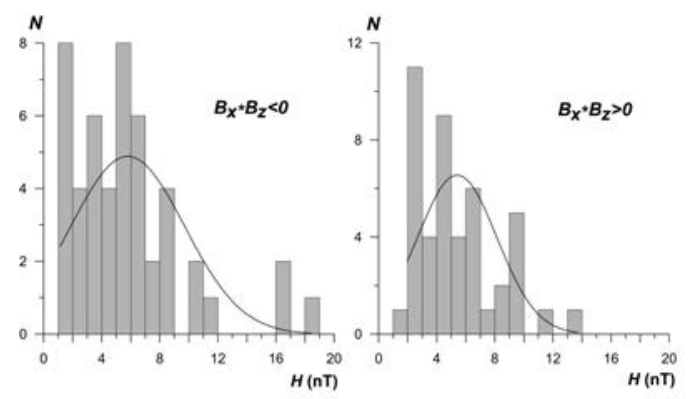

Figure 5. Amplitude distributions of ULF oscillations IPCL

words, we can fairly confidently assert that IPCL activity at Mirny increases when electromagnetic noise originating in a foreshock region affects the southern hemisphere of the magnetosphere.

\section{DISCUSSION}

In our study, we use the classical scheme of "black box with output and input" [Ashby, 1959]. A weak point in our method is that it does not exclude the hypothetical possibility of existence of a self-excited oscillator related to a cusp, which is affected by external noise, randomly changing the mode of self-oscillations. Although we have no evidence of the self-oscillatory origin of IPCLs yet, we should keep this possibility in mind from the principled point of view. Well-known methods of statistical radiophysics allow us to distinguish forced oscillations from self-oscillations. In the past, they were very successfully used for studying Pi1C [Kalisher, 1975] and Pc4 pulsations [Guglielmi, 1989]. IPCLs should be studied in a similar way. In this case, the method of investigating dynamic systems with the scheme of "black box without input" may prove to be effective [Gudzenko, 1962].

Let us turn once more to Figure 3 . It served us as a 
test to check the information content of threedimensional subspaces of the general space of control parameters. The test proved to be satisfactory, although, of course, information on the predominant orientation of IMF lines in the $x-y$ plane during the IPCL observation period is trivial. In view of this, we have indicated above the inequality $\operatorname{sign}\left(B_{x} B_{y}\right)<0$ typical of IMF. It is quite understandable that this inequality imposes no restrictions on the sign of $B_{x}$ that depends on in which IMF sector Earth is located - in positive $\left(B_{X}<0\right)$ or negative $\left(B_{x}>0\right)$. Meanwhile, Figure 3 clearly shows that IPCLs are observed at Mirny mainly at $B_{x}<0$, i.e., when Earth is in the positive sector, and this is surprising. Logically, we must assume that in the Northern Hemisphere, IPCLs will be largely observed when Earth is in the negative IMF sector. We plan to test this assumption, using observational data on ULF oscillations acquired in Greenland.

Apparently, we have unexpectedly discovered another manifestation of the north-south asymmetry of IPCL excitation. Asymmetry of this type is associated not with the orientation of a foreshock relative to the plane of the geomagnetic equator, but with the sign of IMF sector. There arises an interesting problem of interpreting this fact. We do not exclude that the observations suggest a nontrivial mechanism for reconnection of IMF lines with geomagnetic field lines in the vicinity of a cusp.

At the end of this Section, we mention the paper [Guglielmi, Potapov, 2017]. In it, a successful search has been carried out for the north-south asymmetry of Pc1 pulsations in the ionospheric Alfven-Belyaev resonator. Another attempt was inspired by the work [Buchachenko, 2014], in which an idea has been put forward of a possible change in magnetoplastic properties of rocks in the focus of a forthcoming earthquake, with alternating electromagnetic field affecting the focus. IMF orientation, as we have seen, controls the redistribution of electromagnetic energy of very powerful ULF oscillations between the Northern and Southern hemispheres. Hence the idea arose of searching for the north-south asymmetry of global seismic activity. Zotov O.D. undertook a pilot analysis of earthquake catalogs, but found no evidence of the change in the north-south asymmetry of seismic activity with changing IMF orientation [Guglielmi et al., 2017]. But in the course of the study, the author discovered a small but statistically significant increase in seismic activity on the days when Earth crossed boundaries between the IMF sectors. However, this result will be discussed in a separate publication.

\section{CONCLUSION}

We have investigated the north-south asymmetry of ULF oscillations of Earth's electromagnetic field as a function of orientation of IMF lines. As the object of the study, we have chosen powerful oscillations of the IPCL type, observed at Mirny Observatory at near-noon hours under quiet geomagnetic conditions. From geometric considerations, we hypothesized that IPCL activity at Mirny depends on inclination of IMF lines with respect to the plane of the geomagnetic equator (north-south asymmetry). We have proposed a testing procedure which implies that the effect on the northern or southern hemispheres of the magnetosphere can be strengthened or weakened depending on IMF orientation. The result of the study confirmed our hypothesis. This makes plausible the assumption that IPCLs are forced oscillations of a nonlinear dynamical system whose most important structural elements are dayside polar cusps.

We wrote this article in memory of our teacher Professor Valeria Alekseevna Troitskaya to the centenary of her birth. Her outstanding contribution to the study of oscillations of Earth's magnetosphere is widely known. Our study of IPCLs shows that the original methods and approaches of V.A. Troitskaya to the study of oscillations of Earth's electromagnetic field, as well as her fundamental discoveries are relevant to this day. The work was partially carried out with the financial support of Program 15 of the Presidium of the Russian Academy of Sciences and projects of RFBR No. 16-0500056 and 16-05-00631.

\section{REFERENCES}

Ashby W.R. Vvedenie v kibernetiku [Introduction into Cybernetics]. Moscow, IL Publ., 1959, 432 p. (In Russian). English edition: An Introduction to Cybernetics. London, Chapman and Hall LTD, 1957, 303 p.

Bolshakova O.V., Troitskaia V.A., Hessler V.P. On the connection of the position of the polarward boundary of the polar cusp with the intensity of IPCL. Proc. International Symposium on Solar-Terrestrial Physics. Sao Paulo, Brazil, June 17-22, 1974, vol. 2, A75-22580 08-92, pp. 180-184.

Buchachenko A.L. Magnetoplasticity and physics of earthquakes. Can we prevent a catastrophe? Uspekhi phizicheskikh nauk [Physics-Uspekhi]. 2014, vol. 184, no. 1, pp. 101-108. (In Russian).

Davison M. Mnogomernoe shkalirovanie: metody naglyadnogo predstavleniya dannykh [Multidimensional Scaling: Methods of Visual Representation of Data]. Moscow, Finansy i Statistika Publ., 1988, 254 p. (In Russian).

Gudzenko L.I. Statistical method for determining characteristics of non-adjustable self-oscillatory system. Izvestiya vuzov. Radiofizika [Radiophysics and Quantum Electronics]. 1962, vol. 5, no. 3, pp. 572-586. (In Russian).

Guglielmi A.V. MGD-volny v okolozemnoi plazme [MHD Waves in the Near-Earth Plasma]. Moscow, Nauka Publ., 1979, 139 p. (In Russian).

Guglielmi A.V. Ray theory of MHD wave propagation (Overview). Geomagnetizm i aeronomiya [Geomagnetism and Aeronomy]. 1985, vol. 25, no. 3, pp. 356-370. (In Russian).

Guglielmi A.V. Hydromagnetic diagnostics and geoelectric survey. Uspekhi phizicheskikh nauk [Physics-Uspekhi]. 1989, vol. 158, iss. 4, pp. 605-637. (In Russian).

Guglielmi A.V. Ultra-low-frequency electromagnetic waves in the Earth's crust and magnetosphere. Uspekhi phizicheskikh nauk [Physics-Uspekhi]. 2007, vol. 50, no. 12, pp. 1197-1216. (In Russian).

Guglielmi A.V., Pokhotelov O.A. Geoelectromagnetic Waves. Bristol and Philadelphia: IOP Publ. Ltd, 1996. 402 p.

Guglielmi A.V., Potapov A.S. Effect of interplanetary magnetic field on ULF oscillations of ionospheric resonator. Kosmicheskie issledovaniya [Cosmic Research]. 2017, vol. 55, no. 4, pp. 263-267. (In Russian).

Guglielmi A.V., Troitskaya V.A. Geomagnitnye pulsatsii $i$ diagnostika magnitosfery [Geomagnetic Pulsations and Diagnostics of the Magnetosphere]. Moscow, Nauka Publ., 1973, 208 p. (In Russian). 
Guglielmi A.V., Troitskaya V.A. MHD waves in the nearEarth space. Fizika Zemli [Izvestiya, Physics of the Solid Earth]. 1978, no. 10, pp. 95-104. (In Russian).

Guglielmi A.V., Potapov A.S., Dovbnya B.V. Key role of interplanetary magnetic field in forming oscillation regime of the Earth's magnetosphere. Triggernye effekty $v$ geosistemakh: materialy III Vserossiiskogo seminara-soveshchaniya [Trigger Effects in Geosystems: Proc. Third National SeminarWorkshop]. Moscow, June 16-19, 2015. Moscow, GEOS Publ., 2015, pp. 328-334. (In Russian).

Guglielmi A.V., Potapov A.S., Dovbnya B.V., Klain B.I., Zotov O.D., Sterlikova I.V., Ruban V.F., Lavrov I.P. Trigger effects in the magnetosphere: on the $100^{\text {th }}$ anniversary of V.A. Troitskaya. IV Vserossiiskaya konferentsiya "Triggernye effekty v geosistemakh": tezisy dokladoy [IV National Conference "Trigger Effects in Geo-systems": Abstracts]. Moscow, June 6-9, 2017. Moscow, GEOS Publ., 2017, pp. 28-29. (In Russian).

Jacobs J.A. Geomagnetic Micropulsations. New York, Heidelberg, Berlin, Springer-Verlag, 1970, 179 p.

Kalisher A.L. Amplitude distribution function of AA-type geomagnetic pulsations. Geomagnetizm i aeronomiya [Geomagnetism and aeronomy]. 1975, vol. 15, no. 5, pp. 952-953. (In Russian).

Klain B.I., Kurazhkovskaya N.A., Kurazhkovsky A.Yu. Intermittency in wave processes. Fizika Zemli [Izvestiya, Physics of the Solid Earth]. 2008, no. 10, pp. 25-34. (In Russian).

Lundin R., Guglielmi A. Ponderomotive forces in cosmos. Space Sci. Rev. 2006, vol. 127, pp. 1-116.

Nishida A. Geomagnetic diagnosis of the magnetosphere. New York, Heidelberg, Berlin, Springer-Verlag, 1978, 256 p.

Parker E. Dinamicheskie protsessy $v$ mezhplanetnoi srede [Dynamic Processes in Interplanetary Medium]. Moscow, Mir Publ., 1965, 302 p. (In Russian). English edition: Interplanetary Dynamical Processes. Interscience Publishers, 1963, 272 p.

Russell C.T., Hoppe M.M. Upstream waves and particles. Space Sci. Rev. 1983, vol. 34, pp. 115-172.

Troitskaya V.A. Pulsations of the Earth`s electromagnetic field with periods of 1-15 sec and their connection with phenomena in the high atmosphere. J. Geophys. Res. 1961, vol. 66, no. 1, pp. 5-18.
Troitskaya V.A. Classification of rapid pulsations of the magnetic field and Earth currents. Geomagnetizm i aeronomiya [Geomagnetism and Aeronomy]. 1964, vol. 4, pp. 490-495. (In Russian).

Troitskaya V.A. ULF wave investigations in the dayside cusp. Adv. Space Res. 1985, vol. 5, no 4, pp. 219-228.

Troitskaya V.A., Bolshakova O.V. Diurnal latitude variation of the location of the dayside cusp. Planet. Space Sci. 1977, vol. 25, pp. 1167-1169.

Troitskaya V.A., Bolshakova O.V. Diagnostics of the magnetosphere using multipoint measurements of ULF waves. Adv. Space Res. 1988, vol. 8, pp. 413-425.

Troitskaya V.A., Guglielmi A.V. Geomagnetic micropulsations and diagnostics of the magnetosphere. Space Sci. Rev. 1967, vol. 7, no. 5/6, pp. 689-769.

Troitskaya V.A., Guglielmi A.V. Geomagnetic pulsations and diagnostics of the magnetosphere. Uspekhi fizicheskikh nauk [Soviet Physics. Uspekhi]. 1969, vol. 12, no. 2, pp. 195218. (In Russian).

Yamauchi M., Nilsson H., Eliasson L., Norberg O., Boehm M., Clemmons J.H., Lepping R.P., Blomberg L., Ohtani S.-I., Yamamoto T., Mukai T., Terasawa T., Kokubun S. Dynamic response of the cusp morphology to the solar wind: a case study during passage of the solar wind plasma cloud on February 21, 1994. J. Geophys. Res. 1996, vol. 101, pp. 24675-24687.

URL: http://omniweb.gsfc.nasa.gov/ow.html (accessed November 10, 2017).

URL: http://www.wdcb.ru/stp/index.ru.html (accessed November 10, 2017)

How to cite this article

Guglielmi A.V., Klain B.I., Potapov A.S. North-south asymmetry of ultra-low-frequency oscillations of Earth's electromagnetic field. Solar-Terrestrial Physics. 2017. Vol. 3. No. 4. P. 26-31. DOI: 10.12737 / stp-34201703 